\title{
Characterisation of age and polarity at onset in bipolar disorder
}

Janos L. Kalman*, Loes M. Olde Loohuis*, Annabel Vreeker*, Andrew McQuillin, Eli A. Stahl, Douglas Ruderfer, Maria Grigoroiu-Serbanescu, Georgia Panagiotaropoulou, Stephan Ripke, Tim B. Bigdeli, Frederike Stein, Tina Meller, Susanne Meinert, Helena Pelin, Fabian Streit, Sergi Papiol, Mark J. Adams, Rolf Adolfsson, Kristina Adorjan, Ingrid Agartz, Sofie R. Aminoff, Heike Anderson-Schmidt, Ole A. Andreassen, Raffaella Ardau, Jean-Michel Aubry, Ceylan Balaban, Nicholas Bass, Bernhard T. Baune, Frank Bellivier, Antoni Benabarre, Susanne Bengesser, Wade H Berrettini, Marco P. Boks, Evelyn J. Bromet, Katharina Brosch, Monika Budde, William Byerley, Pablo Cervantes, Catina Chillotti, Sven Cichon, Scott R. Clark, Ashley L. Comes, Aiden Corvin, William Coryell, Nick Craddock, David W. Craig, Paul E. Croarkin, Cristiana Cruceanu, Piotr M. Czerski, Nina Dalkner, Udo Dannlowski, Franziska Degenhardt, Maria Del Zompo, J. Raymond DePaulo, Srdjan Djurovic, Howard J. Edenberg, Mariam Al Eissa, Torbjørn Elvsåshagen, Bruno Etain, Ayman H. Fanous, Frederike Fellendorf, Alessia Fiorentino, Andreas J. Forstner, Mark A. Frye, Janice M. Fullerton, Katrin Gade, Julie Garnham, Elliot Gershon, Michael Gill, Fernando S. Goes, Katherine Gordon-Smith, Paul Grof, Jose Guzman-Parra, Tim Hahn, Roland Hasler, Maria Heilbronner, Urs Heilbronner, Stephane Jamain, Esther Jimenez, Ian Jones, Lisa Jones, Lina Jonsson, Rene S. Kahn, John R. Kelsoe, James L. Kennedy, Tilo Kircher, George Kirov, Sarah Kittel-Schneider, Farah Klöhn-Saghatolislam, James A. Knowles, Thorsten M. Kranz, Trine Vik Lagerberg, Mikael Landen, William B. Lawson, Marion Leboyer, Qingqin S. Li, Mario Maj, Dolores Malaspina, Mirko Manchia, Fermin Mayoral, Susan L. McElroy, Melvin G. McInnis, Andrew M. McIntosh, Helena Medeiros, Ingrid Melle, Vihra Milanova, Philip B. Mitchell, Palmiero Monteleone, Alessio Maria Monteleone, Markus M. Nöthen, Tomas Novak, John I. Nurnberger, Niamh O'Brien, Kevin S. O'Connell, Claire O'Donovan, Michael C. O'Donovan, Nils Opel, Abigail Ortiz, Michael J. Owen, Erik Pålsson, Carlos Pato, Michele T. Pato, Joanna Pawlak, Julia-Katharina Pfarr, Claudia Pisanu, James B. Potash, Mark H Rapaport, Daniela Reich-Erkelenz, Andreas Reif, Eva Reininghaus, Jonathan Repple, Hélène RichardLepouriel, Marcella Rietschel, Kai Ringwald, Gloria Roberts, Guy Rouleau, Sabrina Schaupp, William A Scheftner,

Simon Schmitt, Peter R. Schofield, K. Oliver Schubert, Eva C. Schulte, Barbara Schweizer, Fanny Senner, Giovanni Severino, Sally Sharp, Claire Slaney, Olav B. Smeland, Janet L. Sobell, Alessio Squassina, Pavla Stopkova, John Strauss,

Alfonso Tortorella, Gustavo Turecki, Joanna Twarowska-Hauser, Marin Veldic, Eduard Vieta, John B. Vincent, Wei Xu, Clement C. Zai, Peter P. Zandi, Psychiatric Genomics Consortium (PGC) Bipolar Disorder Working Group, International Consortium on Lithium Genetics (ConLiGen), Colombia-US Cross Disorder Collaboration in Psychiatric Genetics, Arianna Di Florio, Jordan W. Smoller, Joanna M. Biernacka, Francis J. McMahon, Martin Alda, Bertram Müller-Myhsok, Nikolaos Koutsouleris, Peter Falkai, Nelson B. Freimer, Till F.M. Andlauert, Thomas G. Schulze† and Roel A. Ophoff†

\section{Background}

Studying phenotypic and genetic characteristics of age at onset (AAO) and polarity at onset (PAO) in bipolar disorder can provide new insights into disease pathology and facilitate the development of screening tools.

\section{Aims}

To examine the genetic architecture of $\mathrm{AAO}$ and PAO and their association with bipolar disorder disease characteristics.

\section{Method}

Genome-wide association studies (GWASs) and polygenic score (PGS) analyses of AAO $(n=12977)$ and PAO $(n=6773)$ were conducted in patients with bipolar disorder from 34 cohorts and a replication sample $(n=2237)$. The association of onset with disease characteristics was investigated in two of these cohorts.

\section{Results}

Earlier AAO was associated with a higher probability of psychotic symptoms, suicidality, lower educational attainment, not living together and fewer episodes. Depressive onset correlated with suicidality and manic onset correlated with delusions and manic episodes. Systematic differences in AAO between cohorts and continents of origin were observed. This was also reflected in single-nucleotide variant-based heritability estimates, with higher heritabilities for stricter onset definitions. Increased PGS for autism spectrum disorder ( $\beta=-0.34$ years, s.e. $=0.08$ ), major depression $(\beta=-0.34$ years, s.e. $=0.08)$, schizophrenia $(\beta=-0.39$ years, s.e. $=$ $0.08)$, and educational attainment $(\beta=-0.31$ years, s.e. $=0.08$ ) were associated with an earlier AAO. The AAO GWAS identified one significant locus, but this finding did not replicate. Neither GWAS nor PGS analyses yielded significant associations with PAO.

\section{Conclusions}

AAO and PAO are associated with indicators of bipolar disorder severity. Individuals with an earlier onset show an increased polygenic liability for a broad spectrum of psychiatric traits. Systematic differences in AAO across cohorts, continents and phenotype definitions introduce significant heterogeneity, affecting analyses.

\section{Keywords}

Bipolar disorder; age at onset; polarity at onset; GWAS; polygenic score.

\section{Copyright and usage}

(c) The Author(s), 2021. Published by Cambridge University Press on behalf of the Royal College of Psychiatrists. This is an Open Access article, distributed under the terms of the Creative Commons Attribution licence (http://creativecommons.org/ licenses/by/4.0/), which permits unrestricted re-use, distribution, and reproduction in any medium, provided the original work is properly cited.
* Joint first authors.

† Joint last authors.

\section{Background}

Bipolar disorder is highly heritable and affects approximately $1 \%$ of the population. It has a recurrent or chronic course and is associated with psychosocial impairment and reduced functioning, and it is a 
leading cause of global disease burden. ${ }^{1}$ Individuals usually experience their first (hypo)manic or depressive episode of bipolar disorder in adolescence or early adulthood, but often they are not diagnosed until 5 to 10 years later, ${ }^{2}$ especially in individuals with an earlier age at onset (AAO) or a depressive index episode. ${ }^{3}$ Early illness onset is associated with a more severe disease course and greater impairment across a wide range of mental and physical disorders and is a useful prognostic marker. ${ }^{4-7}$ However, pathophysiological processes leading to a disorder are thought to begin long before the first symptoms appear. ${ }^{8,9}$ Investigating the factors contributing to age and polarity (i.e. either a (hypo)manic or depressive episode) at onset could thus improve our understanding of disease pathophysiology and facilitate development of personalised screening and preventive measures. Accordingly, AAO and polarity at onset (PAO) of bipolar disorder are considered as suitable phenotypes for genetic analyses.

Genome-wide association studies (GWASs) have improved our understanding of the genetic architecture of susceptibility to bipolar disorder; however, the genetic determinants of AAO and PAO remain largely unknown. Evidence suggests that patients with an early AAO carry a stronger genetic loading for bipolar disorder risk. ${ }^{10}$ For example, an earlier parental AAO increases familial risk for bipolar disorder and is one of the strongest predictors of 5-year illness onset in affected offspring. ${ }^{10-12}$ Previous research has described that a higher genetic risk burden for schizophrenia may be associated with earlier AAO of bipolar disorder, ${ }^{13}$ but this finding did not replicate. ${ }^{14-16}$ Moreover, a recent study did not find an association of bipolar disorder polygenic score (PGS) with AAO. ${ }^{17}$ Thus far, GWASs for age at bipolar disorder onset have been underpowered, ${ }^{18,19}$ and a study of 8610 patients found no significant evidence for a heritable component contributing to onset age. ${ }^{13}$ The PAO was shown to cluster in families, ${ }^{20}$ but the genetic architecture of PAO has not yet been investigated.

\section{Aims}

To fill these knowledge gaps, we performed comprehensive analyses of AAO and PAO of bipolar disorder in the largest sample studied to date by (a) examining phenotype definitions and associations, (b) investigating whether the genetic load for neuropsychiatric disorders and traits contributes to $\mathrm{AAO}$ and $\mathrm{PAO}$ of bipolar disorder, and (c) conducting systematic GWASs.

\section{Method}

References to published methods are listed in Supplementary Note 1 available at https://doi.org/10.1192/bjp.2021.102.

\section{Study samples}

Participants with a bipolar disorder diagnosis, available genetic data and AAO information were selected from independent data-sets, including those previously submitted to the Psychiatric Genomics Consortium (PGC) Bipolar Disorder Working Group ${ }^{13}$ and the International Consortium on Lithium Genetics (ConLiGen). ${ }^{21}$ These consortia aggregate genetic data from many cohorts worldwide. Our analyses comprised 34 cohorts with 12977 patients with bipolar disorder who have European ancestry from Europe, North America and Australia. For a description of sample ascertainment, see the Supplementary Material.

The authors assert that all procedures contributing to this work comply with the ethical standards of the relevant national and institutional committees on human experimentation and with the Helsinki Declaration of 1975, as revised in 2008. All procedures involving human patients were approved by the local ethics committees, and written informed consent was obtained from all patients. For details on the data-sets, including phenotype definitions and distributions, see Table 1, Fig. 1, and Supplementary Table S1.

\section{Definition of AAO}

The definition of age at bipolar disorder onset differed by cohort. To enhance cross-cohort comparability, we grouped the definitions into four broad categories as follows (Supplementary Table S1).

(a) Diagnostic interview: age at which the patient first experienced a (hypo)manic, mixed or major depressive episode according to a standardised diagnostic interview.

(b) Impairment/help-seeking: age at which symptoms began to cause subjective distress or impaired functioning or at which the patient first sought psychiatric treatment.

(c) Pharmacotherapy: age at first administration of medication.

(d) Mixed: a combination of the above-mentioned definitions.

Across definitions, participants younger than 8 years at onset were excluded ( $n=279$ ) because of the uncertainty about the reliability of retrospective recall of early childhood onset. The distribution of AAO was highly skewed and differed considerably between the cohorts (Table 1 and Fig. 1). Therefore, we transformed AAO in each cohort by rank-based inverse-normal transformation and used this normalised variable as the primary dependent variable in all genetic analyses. To facilitate interpretability of effect sizes, we also report results of the corresponding untransformed AAO.

\section{Definition of PAO}

For each cohort, PAO was defined by comparing the age at the first (hypo)manic and first depressive episode or using the polarity variable provided by the cohort. Specifically, patients were divided into three subgroups:

(a) (hypo)mania before depression (PAO-M);

(b) depression before (hypo)mania (PAO-D); and

(c) mixed (PAO-X).

The third category included patients with mixed episodes and those with a first (hypo)manic and depressive episode within the same year (Table 1). In the primary analysis, we combined patients with (hypo)mania and mixed onset and assigned this as the reference category. In secondary analyses, we excluded the patients in the mixed group.

\section{Phenotypic disease characteristics}

We performed phenotypic analyses of disease onset in patients with bipolar disorder type I from three cohorts: the Dutch Bipolar cohort $(n=1313)^{22}$ and the German PsyCourse ${ }^{23}$ and FOR $2107^{24}$ cohorts, which were analysed jointly $(n=346)$. We analysed the following disease characteristics, which were previously reported as being associated with disease onset and were assessed in a similar way across cohorts: lifetime delusions, lifetime hallucinations, history of suicide attempt, suicidal ideation, current smoking, educational attainment, living together with a partner, and frequency of manic and depressive episodes per year. For more detailed information, see the Supplementary Note 2 and Supplementary Table S9.

\section{Quality control and imputation of genotype data}

The cohorts were genotyped according to local protocols. Individual genotype data of all discovery-stage cohorts were processed with the PGC Rapid Imputation and Computational Pipeline for GWAS (RICOPILI) with the default parameters for standardised quality control, imputation and analysis. Before imputation, filters for the removal of variants included non-autosomal chromosomes, missingness $\geq 0.02$, and a Hardy-Weinberg equilibrium test $P<1 \times 10^{-10}$. 
Table 1 Sample characteristics of data-sets used in genetic analyses

\begin{tabular}{|c|c|c|c|c|c|c|c|}
\hline GWAS stage, dataset & $n$ & Continent & Diagnosis, \% bipolar disorder type I & Gender, \% male & AAO, median ( $M A D,{ }^{a}$ range) & Definition of AAO & $\mathrm{PAO}^{\mathrm{b}} n(\%)$ \\
\hline \multicolumn{8}{|l|}{ Discovery } \\
\hline wtccc & 1452 & Europe & 89.53 & 36.85 & $24(8.9,9-63)$ & Impairment/help-seeking & \\
\hline $\operatorname{tgco2}$ & 865 & North America & 100 & 33.64 & $17(5.93,8-46)$ & Diagnostic interview & PAO-M: 316 (38.92); PAO-D: 496 (61.08) \\
\hline gain & 797 & North America & 100 & 48.06 & $18(5.93,8-45)$ & Diagnostic interview & PAO-M: 135 (18.57); PAO-D: 440 (60.52) \\
\hline $\operatorname{stp} 1$ & 718 & North America & 100 & 44.01 & $16(5.93,8-41)$ & Diagnostic interview & PAO-M: 137 (19.08); PAO-D: 420 (58.5) \\
\hline gsk1 & 715 & North America & 89.51 & 36.36 & $19(7.51,8-52)$ & Diagnostic interview & PAO-M: 102 (14.61); PAO-D: 395 (56.59) \\
\hline usc2 & 681 & North America & 96.18 & 47.58 & $18(7.41,8-48)$ & Impairment/help-seeking & \\
\hline bonn & 638 & Europe & 99.84 & 47.34 & $25(8.9,9-64)$ & Impairment/help-seeking & \\
\hline ucl2 & 604 & Europe & 100 & 44.37 & $30(11.86,9-60)$ & Pharmacotherapy & PAO-M: 47 (9.96); PAO-D: 209 (44.28) \\
\hline bmg3 & 455 & Europe & 57.14 & 40.66 & $24(10.38,10-62)$ & Impairment/help-seeking & PAO-M: 43 (16.35); PAO-D: 159 (60.46) \\
\hline m\&m's & 449 & Europe & 74.83 & 52.12 & $23(10.38,8-65)$ & Mixed & PAO-M: 73 (17.14); PAO-D: 238 (55.87) \\
\hline uclo & 439 & Europe & 100 & 39.86 & $22(7.41,8-51)$ & Impairment/help-seeking & PAO-M: 54 (14.25); PAO-D: 197 (51.98) \\
\hline fran & 411 & Europe & 77.62 & 41.36 & $22(7.41,10-58)$ & Diagnostic interview & \\
\hline euor & 410 & Europe & 75.85 & 44.15 & $22(9.64,11-59)$ & Mixed & \\
\hline hal2 & 355 & North America & 71.55 & 42.54 & $23(8.9,8-56)$ & Diagnostic interview & PAO-M: 102 (29.65); PAO-D: 213 (61.92) \\
\hline ume4 & 354 & Europe & 69.21 & 37.85 & $20(8.9,8-63)$ & Diagnostic interview & PAO-M: 54 (14.25); PAO-D: 197 (51.98) \\
\hline swa2 & 344 & Europe & 81.10 & 41.86 & $23(10.38,10-70)$ & Impairment/help-seeking & \\
\hline bmpo & 319 & Europe & 78.06 & 39.18 & $28(11.86,10-63)$ & Impairment/help-seeking & PAO-M: 41 (16.33); PAO-D: 150 (59.76) \\
\hline top7 & 301 & Europe & 62.79 & 41.53 & $19(7.41,8-49)$ & Diagnostic interview & \\
\hline may1 & 257 & North America & 100 & 45.14 & $20(8.9,8-62)$ & Diagnostic interview & PAO-M: 34 (13.23); PAO-D: 142 (55.25) \\
\hline bmsp & 248 & Europe & 94.76 & 45.56 & $22(7.41,9-57)$ & Impairment/help-seeking & PAO-M: 24 (10.04); PAO-D: 93 (38.91) \\
\hline bmau & 245 & Australia & 79.18 & 40.82 & $19(7.41,8-55)$ & Diagnostic interview & PAO-M: 46 (20.18); PAO-D: 125 (54.82) \\
\hline edi1 & 244 & Europe & 99.18 & 42.62 & $20(5.93,13-50)$ & Diagnostic interview & \\
\hline rom3 & 226 & Europe & 100 & 41.15 & $25(10.38,12-59)$ & Diagnostic interview & PAO-M: 91 (40.27); PAO-D: 134 (59.29) \\
\hline butr & 204 & Europe & 100 & 40.2 & $22(5.19,13-44)$ & Impairment/help-seeking & \\
\hline enol & 191 & Europe & 74.87 & 31.41 & $24(8.9,13-67)$ & Diagnostic interview & PAO-M: 48 (27.43); PAO-D: 98 (56) \\
\hline ageu & 178 & Europe & 90.45 & 39.33 & $21(7.41,8-51)$ & Impairment/help-seeking & \\
\hline mich & 169 & North America & 100 & 31.36 & $18(5.93,8-45)$ & Diagnostic interview & PAO-M: 42 (24.85); PAO-D: 84 (49.7) \\
\hline naom & 159 & North America & 84.91 & 44.65 & $18(7.41,8-66)$ & Mixed & PAO-M: 30 (28.85); PAO-D: 51 (49.04) \\
\hline bmg2 & 152 & Europe & 59.87 & 35.53 & $27(10.38,13-63)$ & Impairment/help-seeking & \\
\hline top8 & 111 & Europe & 55.86 & 37.84 & $18(7.41,8-49)$ & Diagnostic interview & \\
\hline h66x & 92 & Europe & 82.61 & 36.96 & $30(10.38,9-55)$ & Mixed & \\
\hline auom & 85 & Australia & 88.24 & 45.88 & $25(10.38,8-64)$ & Diagnostic interview & \\
\hline euo2 & 58 & Europe & 65.52 & 56.9 & $26(8.9,18-57)$ & Diagnostic interview & \\
\hline dub1 & 51 & Europe & 100 & 54.9 & $21(5.93,12-45)$ & Diagnostic interview & \\
\hline Summary & 12977 & & 88.27 & 41.57 & $21(8.9,8-70)$ & & PAO-M: 1435 (21.19); PAO-D: 3885 (57.36) \\
\hline \multicolumn{8}{|l|}{ Replication } \\
\hline ukwa1 & 1156 & Europe & 75.17 & 38.15 & $23(8.9,8-74)$ & Impairment/help-seeking & \\
\hline dutch & 468 & Europe & 100 & 42.31 & $28(10.38,11-63)$ & Pharmacotherapy & \\
\hline jst5 & 186 & North America & 100 & 53.23 & $16(7.41,8-51)$ & Unknown & \\
\hline colo & 176 & South America & 90.34 & 31.82 & $20(11.86,8-52)$ & Diagnostic interview & \\
\hline bmrom & 126 & Europe & 100 & 42.86 & $24(8.9,12-56)$ & Diagnostic criteria & \\
\hline bdtrs & 125 & Europe & 64 & 45.6 & $28(13.34,8-65)$ & Impairment/help-seeking & \\
\hline Summary & 2237 & & 84.40 & 40.46 & $24(10.38,8-74)$ & & \\
\hline All data & 15214 & & 86.26 & 41.41 & $22(8.9,8-74)$ & & \\
\hline
\end{tabular}


(a)

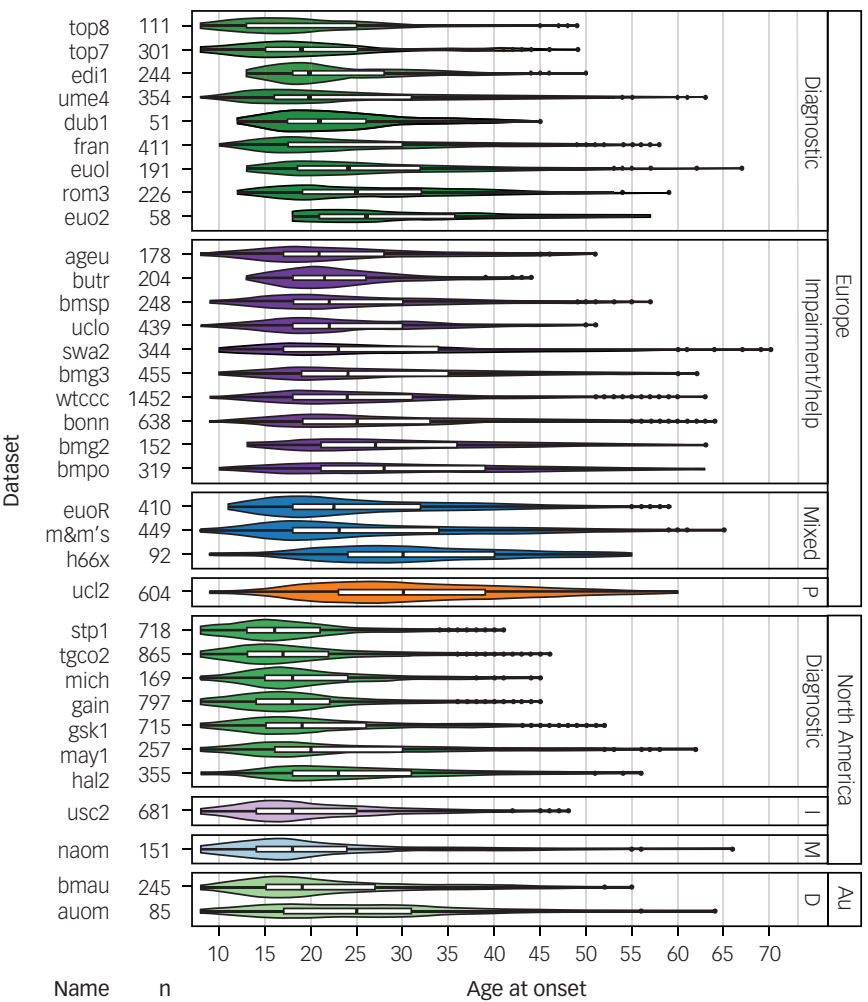

(b)

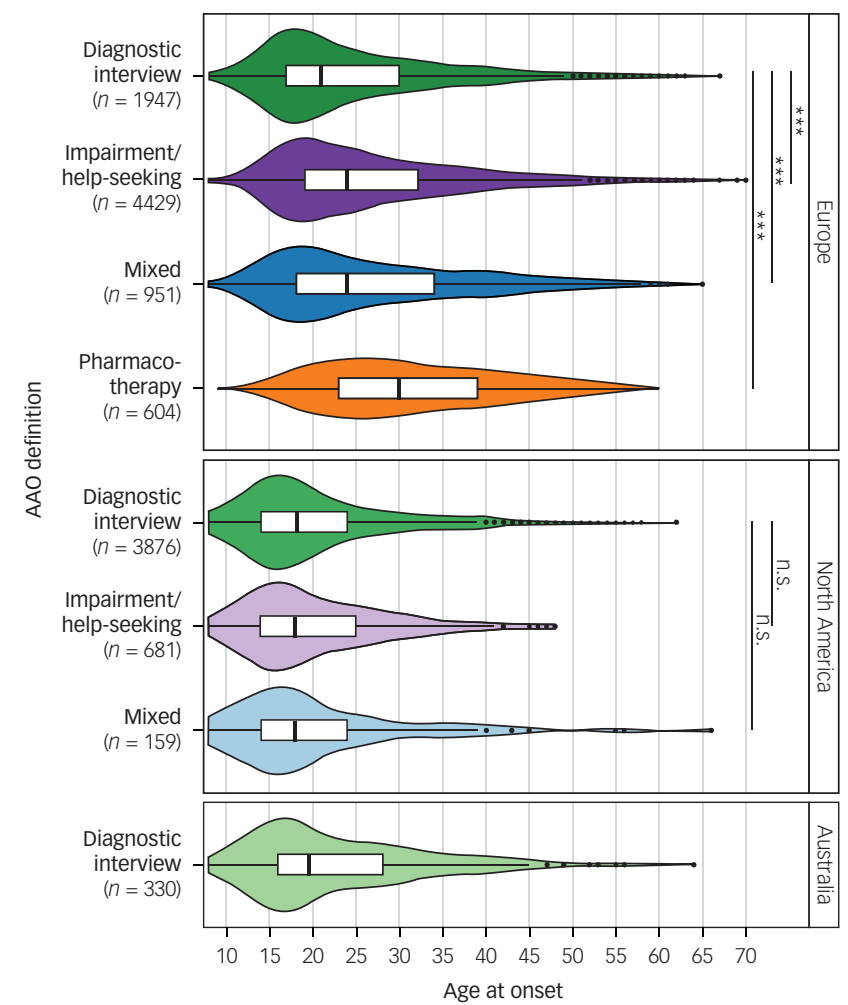

\section{Fig. 1 Differences between phenotype definitions and continents across the 34 data-sets used for discovery-stage genetic analyses.}

(a) The various data-sets used four different definitions for age at onset: diagnostic interview, impairment/help-seeking, pharmacotherapy and mixed. (b) The untransformed age at onset differed significantly between cohorts, depending on the phenotype definition used and the continent of origin.

$\mathrm{Au}$, Australia; Diagnostic, diagnostic interview; $\mathrm{D}$, diagnostic interview; I, impairment/help-seeking; $\mathrm{M}$, mixed; $P$, pharmacotherapy. $n . S$., not significant; $P>0.05$; $* \star * ~ P<0.001$.

Individuals were removed if they showed a genotyping rate $\leq 0.98$, absolute deviation in autosomal heterozygosity of $F_{\text {het }} \geq 0.2$, or a deviation $>4$ s.d.s from the mean in any of the first eight ancestry components within each cohort. From genetic duplicates and relatives (pihat $>0.2$ ) across all samples, only the individual with more complete phenotypic information on AAO and PAO, gender and diagnosis was retained. Imputation was performed by IMPUTE2 with the Haplotype Reference Consortium reference panel.

\section{PGS}

We calculated PGS based on prior GWAS of attention-deficit hyperactivity disorder (ADHD), autism spectrum disorder (ASD), bipolar disorder, educational attainment (measured as 'years in education'), major depression (MD), and schizophrenia (see Supplementary Table S3, which includes references). PGS weights were estimated with PRS-CS(see Supplement), with six scores per GWAS (with $\varphi$ $=1 \times 10^{-1}, 1 \times 10^{-2}, 1 \times 10^{-3}, 1 \times 10^{-4}, 1 \times 10^{-5}$, and $\left.1 \times 10^{-6}\right)$. We tested the associations of the PGS with the AAO and PAO by linear and logistic regressions, respectively. Gender, bipolar disorder subtype and the first eight ancestry components were included as covariates. The significance threshold was Bonferroni-corrected for 96 tests $(\alpha=0.05 /(6 \varphi$ thresholds $\times 8$ traits $\times 2$ phenotypes $)=$ $\left.5.2 \times 10^{-4}\right)$.

\section{GWASS}

We performed a discovery GWAS on the 34 cohorts $(n=12977)$ and replication analyses in six additional cohorts with $n=2237$ patients with bipolar disorder. As a first step, we conducted individual GWAS for each cohort with 40 or more patients using the
RICOPILI workflow, using the same covariates as in the PGS analyses. Sample sizes are provided in Supplementary Tables S2 and S7. The resulting GWAS did not show an inflation of test statistics for any of the cohorts, indicating limited population stratification (Supplementary Table S2). Next, we performed a fixed-effects meta-analysis using METAL, combining the cohort-specific GWASs. For the meta-analysis summary statistics, we applied the following variant-level post-quality control parameters: imputation INFO score $\geq 0.9$, minor allele frequency (MAF) $\geq 0.05$, and successfully imputed/genotyped in more than half of the cohorts.

The primary analyses were AAO (normalised, analysed by linear regression) and PAO (analysed by logistic regression). Secondary analyses included GWASs stratified by AAO definition and continent of origin.

We estimated the power to replicate our initial genome-wide significant finding from the discovery GWAS based on the regression coefficients using the $p w r$ package in $R$. Assuming the same effect size and MAF (beta 0.075 , allele frequency 0.32 ) and a standardised phenotype, we had $76 \%$ power to detect the effect in our sample size of 2237 at an alpha level of 0.1 . For comparison, we had $57 \%$ power to detect the effect in our discovery sample, using the more stringent genome-wide significance cut-off.

\section{Heritability analyses}

Next, we assessed the overall variance in AAO and PAO explained by genotyped variants (so-called single-nucleotide variant (SNV)based heritability, $h_{S N V}^{2}$ ). For the only individual cohort with more than 1000 samples, we estimated $h_{S N V}^{2}$ with GCTA GREML. In this case, we validated the robustness of the $h_{S N V}^{2}$ estimate with 
Table 2 The association of age and polarity at onset with disease characteristics in two European bipolar disorder cohorts

\begin{tabular}{|c|c|c|c|c|c|c|c|c|c|c|}
\hline \multirow[b]{2}{*}{ Disease characteristic } & \multicolumn{5}{|c|}{ AAO } & \multicolumn{5}{|c|}{ PAO } \\
\hline & $n$ & Odds ratio & $95 \% \mathrm{Cl}$ & Unadjusted $P$ & Adjusted $P^{a}$ & $n$ & Odds ratio & $95 \% \mathrm{Cl}$ & Unadjusted $P$ & Adjusted $P$ \\
\hline Delusions & 1612 & 0.71 & $0.64-0.79$ & $1.61 \times 10^{-9}$ & $1.45 \times 10^{-8^{*}}$ & 1298 & 0.62 & $0.49-0.79$ & $1.04 \times 10^{-4}$ & $6.24 \times 10^{-4^{*}}$ \\
\hline Hallucinations & 1594 & 0.83 & $0.74-0.92$ & $3.5 \times 10^{-4}$ & $1.40 \times 10^{-3^{*}}$ & 1290 & 0.93 & $0.74-1.17$ & $5.22 \times 10^{-1}$ & $1.00 \times 10^{0}$ \\
\hline Current smoking & 1594 & 0.98 & $0.89-1.09$ & $7.50 \times 10^{-1}$ & $7.50 \times 10^{-1}$ & 1282 & 1.12 & $0.89-1.41$ & $3.39 \times 10^{-1}$ & $1.00 \times 10^{0}$ \\
\hline Suicidal ideation & 1518 & 0.79 & $0.71-0.88$ & $2.31 \times 10^{-5}$ & $1.62 \times 10^{-4^{*}}$ & 1280 & 1.68 & $1.32-2.13$ & $2.11 \times 10^{-5}$ & $1.48 \times 10^{-4^{*}}$ \\
\hline Suicide attempt & 1537 & 0.78 & $0.69-0.88$ & $2.73 \times 10^{-5}$ & $1.64 \times 10^{-4^{*}}$ & 1262 & 1.58 & $1.24-2.02$ & $2.67 \times 10^{-4}$ & $1.34 \times 10^{-3^{*}}$ \\
\hline Educational attainment & 1636 & 1.17 & $1.06-1.29$ & $2.77 \times 10^{-3}$ & $8.31 \times 10^{-3^{*}}$ & 1319 & 1.06 & $0.85-1.33$ & $5.93 \times 10^{-1}$ & $1.00 \times 10^{0}$ \\
\hline Living together & 1357 & 1.28 & $1.15-1.44$ & $1.01 \times 10^{-5}$ & $8.08 \times 10^{-5^{*}}$ & - & - & - & - & - \\
\hline
\end{tabular}

the mean of $1000 \times$ resampling of $95 \%$ of the sample. To estimate the overall heritability of the meta-analysis summary statistics we estimated $h_{S N V}^{2}$ by linkage disequilibrium score regression, for each GWAS with sample size $>3000$. The $95 \%$ CIs were constrained to a minimum of 0 and a maximum of 1 .

\section{Results}

\section{Heterogeneity of AAO and PAO across cohorts}

Among the four definitions of AAO across the 34 cohorts, impairment/help-seeking was the most common in Europe and diagnostic interview the most common in North America (Table 1, Fig. 1). Across all cohorts, the median AAO was 21 years (range of medians: 16-30 years; Fig. 1). However, substantial differences in the AAO were observed between subgroups: first, the median untransformed AAO was lower in bipolar disorder type I than in type II (type I, 21 years; type II, 22 years; Kruskal-Wallis test $P=1.8 \times 10^{-4}$; Supplementary Table S6).

Second, the AAO was lower when determined by diagnostic interview compared with other phenotype definitions (diagnostic interview, 19 years; impairment/help-seeking, 23 years; pharmacotherapy, 30 years; mixed, 22 years; $P=2.96 \times 10^{-191}$ ). Third, the age was lower in North America compared with Europe (Europe, 24 years; North America, 18 years; and Australia, 19.5 years; $P=$ $2.0 \times 10^{-263}$ ). These differences across continents remained significant when including onset definitions and bipolar disorder subtype in a multivariable regression model, indicating that they are likely partially independent from the assessment strategy (Supplementary Table S6).

The majority of patients reported a depression-first PAO. Patients with depression-first were less frequent in the impairment/help-seeking than in the diagnostic interview category $(55 \%$ and $60 \%$, respectively; $P=4.5 \times 10^{-4}$, Supplementary Fig. S1), but their proportions were similar between Europe and North America (57\% and 59\%, respectively; $P=0.17$ test of proportion).

\section{Analyses of disease characteristics}

In a meta-analysis of the Dutch and German samples, earlier AAO was significantly associated with a higher probability of lifetime delusions, hallucinations, suicide attempts, suicidal ideation, lower educational attainment and not living together (Table 2, Supplementary Tables S4 and S5). A later AAO was positively significantly correlated with a higher number of manic and depressive episodes per year (see Tables 3, and the Supplementary Note 2). Moreover, a (hypo)manic onset was significantly associated with a greater likelihood of delusions and more manic episodes per year, whereas a depressive onset was associated with a higher probability of suicidal ideation and lifetime suicide attempts.

\section{Associations of PGSs with AAO and PAO}

Next, we conducted analyses to evaluate whether the genetic liability for five psychiatric disorders and educational attainment were associated with the age at disease onset (Fig. 2(a) and (b) and Supplementary Table S8). After correcting for 96 tests, higher PGSs for ASD ( $\beta=-0.34$ years per 1 s.d. increase in PGS, s.e. $=$ $\left.0.08, P=9.85 \times 10^{-6}\right)$, major depression $(\beta=-0.34$, s.e. $=0.08, P=$ $\left.1.40 \times 10^{-6}\right)$, schizophrenia $\left(\beta=-0.39\right.$, s.e. $\left.=0.08, P=2.91 \times 10^{-6}\right)$ and educational attainment $\left(\beta=-0.31\right.$, s.e. $\left.=0.08, P=5.58 \times 10^{-5}\right)$ were significantly associated with an earlier age at bipolar disorder onset. This was not the case for ADHD or bipolar disorder PGS. No PGS was significantly associated with PAO (Supplementary Fig. S4, Supplementary Table S8).

\section{GWASS}

Next, we attempted to identify individual genetic loci associated with the AAO or PAO. In our discovery GWAS using 34 cohorts, one locus was significantly associated with AAO (rs1610275 on chromosome 16; minor allele $G$ frequency $=0.319, \beta=0.075$ (s.e. $=0.014$ ), $P=3.39 \times 10^{-8}$, Fig. 2(c), Supplementary Table S7, Supplementary Fig. S2). This SNV mapped to an intron of the brain-expressed gene FTO (alpha-ketoglutarate dependent dioxygenase, Fig. 2(d)).

\begin{tabular}{|c|c|c|c|c|c|c|c|c|c|c|}
\hline \multirow[b]{2}{*}{ Episode } & \multicolumn{5}{|c|}{ AAO } & \multicolumn{5}{|c|}{ PAO } \\
\hline & \multicolumn{4}{|r|}{ Unadjusted } & Adjusted $P^{c}$ & \multicolumn{5}{|c|}{ Unadjusted } \\
\hline $\begin{array}{l}\text { Number of manic episodes per illness } \\
\text { year }\end{array}$ & 1436 & 0.11 & 0.03 & $7.08 \times 10^{-5}$ & $3.54 \times 10^{-4^{*}}$ & 1156 & -0.42 & 0.06 & $4.68 \times 10^{-13}$ & $3.74 \times 10^{-12^{*}}$ \\
\hline $\begin{array}{l}\text { Number of depressive episodes per } \\
\text { illness year }\end{array}$ & 1231 & 0.07 & 0.03 & $1.93 \times 10^{-2}$ & $3.86 \times 10^{-2^{*}}$ & 1051 & 0.12 & 0.06 & $4.63 \times 10^{-2}$ & $1.85 \times 10^{-1}$ \\
\hline $\begin{array}{l}\text { AAO, age at onset; } P A O \text {, polarity at onset; } n \text {, to } \\
{ }^{*} P<0.05 \\
\text { a. The number of manic/depressive episodes } \\
\text { Supplementary Note } 2 \text {. } \\
\text { b. Unstandardised beta coefficient. } \\
\text { c. After Bonferroni-Holm correction. }\end{array}$ & numb & $\begin{array}{l}\text { f participan } \\
\text { y (years of }\end{array}$ & fich & $\begin{array}{l}\text { Dutch and Ger } \\
\text { Eor secondary }\end{array}$ & $\begin{array}{l}\text { cohorts. } \\
\text { yses of the nu }\end{array}$ & $f$ & tes net & ter & e years of ill & see the \\
\hline
\end{tabular}


(a)
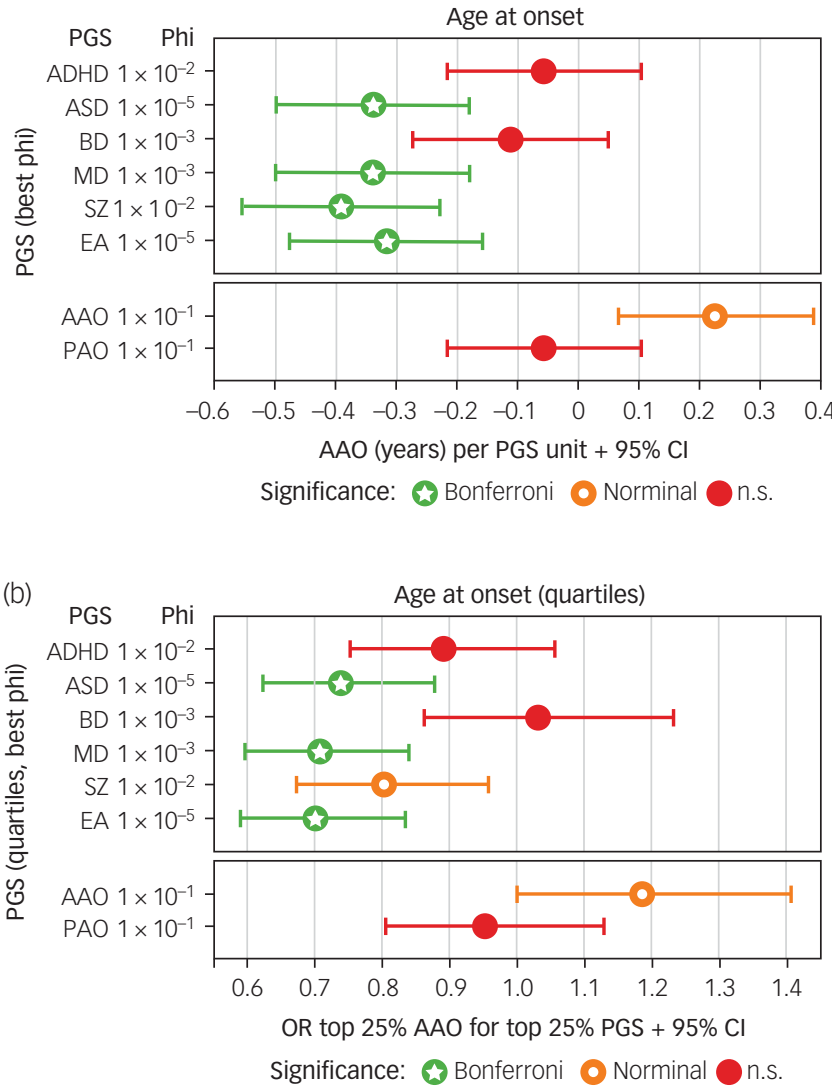

(c)

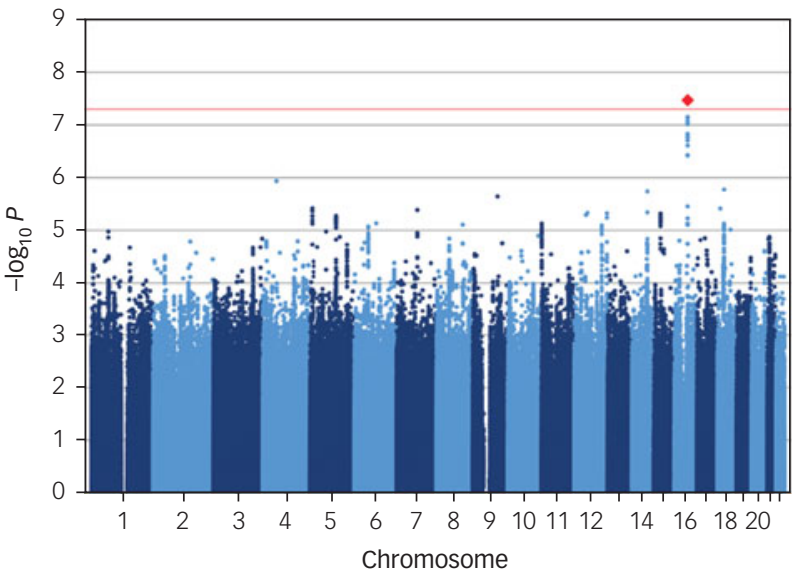

(d)

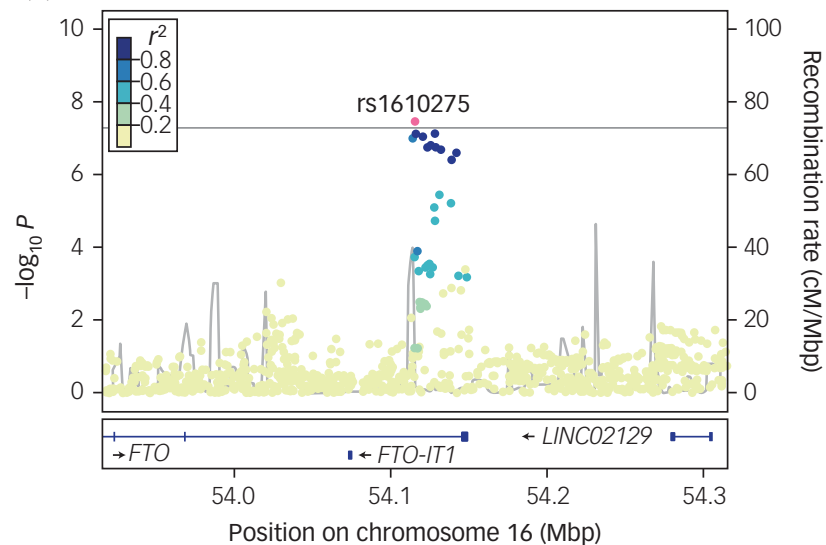

(e)

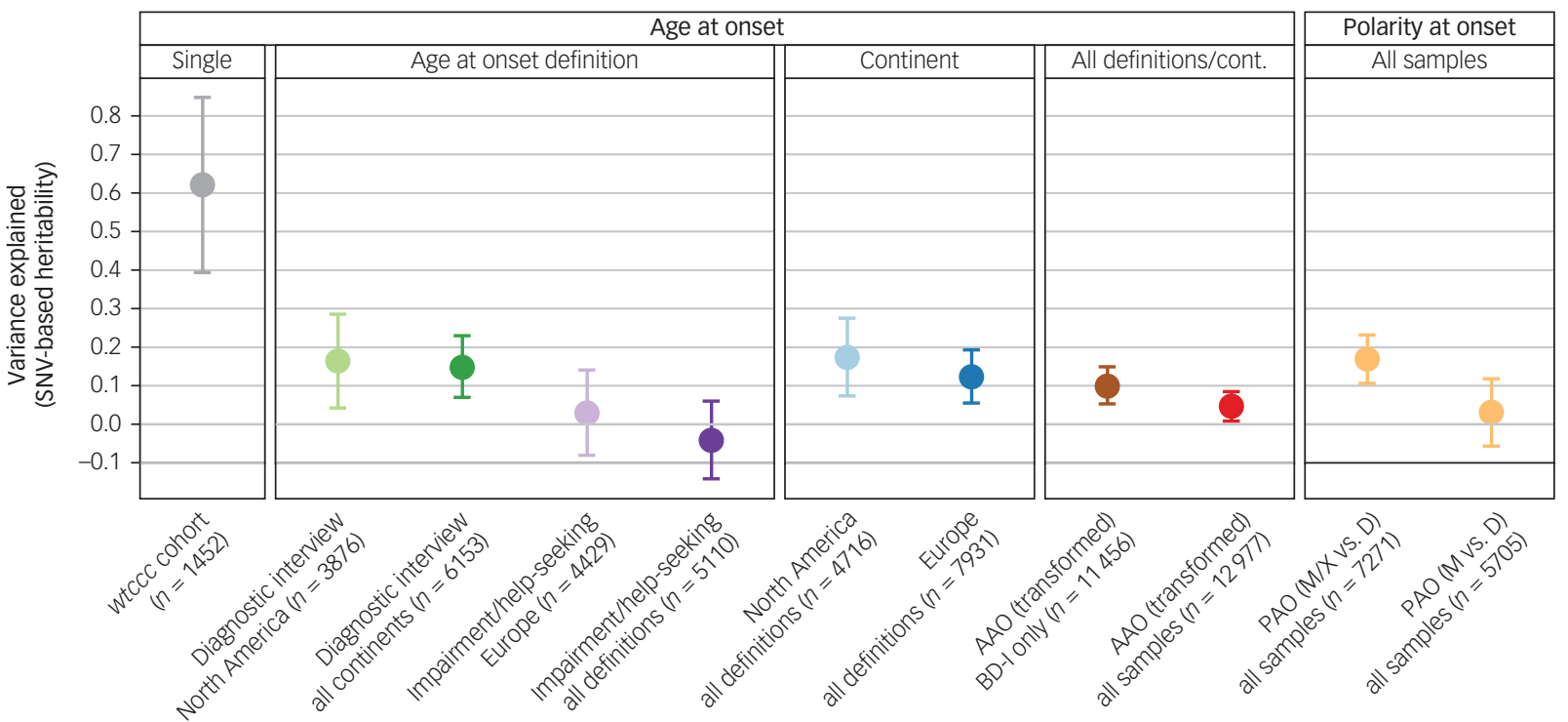

\section{Fig. 2 Results from the genome-wide association study (GWAS), polygenic score (PGS) analyses, and heritability analyses.}

(a) and (b) Results from analyses of PGS. For detailed results, see Supplementary Table S8. Significance levels: $n . S$. , not significant, $P>0.05$; nominal: $P<0.05$; Bonferroni, below the Bonferroni-corrected significance threshold corrected for 96 tests $\left(P<5.2 \times 10^{-4}\right)$. (a) Associations of PGSs with the AAO. For interpretability, the plot shows the untransformed AAO. Significance levels are based on the analyses of the AAO after rank-based inverse-normal transformation (which was performed because the distribution of AAO was highly skewed and differed greatly across the study cohorts). (b) Associations of the top versus bottom AAO quartiles with the top versus bottom PGS quartiles. A higher odds ratio (OR) indicates an association with higher AAO. (c) Manhattan plot of the discovery-stage AAO GWAS. (d) Locus-specific Manhattan plot of the top-associated AAO variant. (e) Estimation of the variance in different phenotype definitions explained by genotyped single-nucleotide variants (SNV) $\left(h_{S N V)}^{2}\right.$ ). For the cohort wtccc, we directly estimated $h_{S N V}^{2}$ from genotype data in GCTA GREML; we estimated all other heritabilities from GWAS summary statistics using LDSC. The plot shows $h_{S N V}^{2}$ estimates and s.e.

ADHD, attention-deficit/hyperactivity disorder; ASD, autism spectrum disorder; BD, bipolar disorder; $C M$, centi Morgan. Mbp, mega base pairs; MD, major depression; EA, educational attainment; SNV, single-nucleotide variant; cont, continent; disorder type I; PAO, polarity at onset; PAO-M, mania/hypomania before depression; PAO-D, depression before mania/hypomania; PAO-X, mixed; SZ, schizophrenia. 
However, this association was not replicated in an independent sample of six cohorts (Supplementary Table S7, Supplementary Fig. S2). In the replication sample $(n=2237)$, we had $76 \%$ power to replicate this SNV at a $P$-value threshold of 0.1. The GWAS of $\mathrm{PAO}$ did not yield any genome-wide significant findings, in either primary (PAO-M/-X versus PAO-D) or secondary (PAO-M versus PAO-D) analyses (Supplementary Fig. S3).

We also calculated PGSs for AAO and PAO using leave-one-out summary statistics from these GWASs. The AAO PGS was nominally significantly associated with AAO $(\beta=0.23$ years, s.e. $=0.08$, $P=0.0087, \varphi=0.1$, Fig. 2(a) and 2(b)) for five of six tested $\varphi$ parameters but did not withstand correction for multiple testing (Supplementary Table S8). The PAO PGS was not associated with the PAO (Supplementary Fig. S4).

\section{SNV-based heritability of the investigated phenotypes}

We estimated the SNV-based heritability $h_{S N V}^{2}$ directly from genotype data using GCTA in the only cohort large enough for this analysis, $w t c c c$. For the AAO, the $h_{S N V}^{2}$ in $w t c c c$ was estimated at $0.63(P=$ 0.0026 ) (Fig. 2(e)). We evaluated the robustness of this estimate by resampling (mean $h_{S N V}^{2}=0.62$, resampling 95\% CI 0.15-1.00).

We next estimated $h_{S N V}^{2}$ by linkage disequilibrium score regression (LDSC) from the GWAS summary statistics generated in the present study (Fig. 2(e)). We observed that the heritability decreased when cohorts, phenotype definitions and continents were combined (for example 'diagnostic interview' in North America: $\mathrm{AAO} \quad h_{S N V}^{2}=0.16,95 \%$ CI 0-0.40, 'impairment/help-seeking' in Europe: $h_{S N V}^{2}=0.03,95 \%$ CI $0-0.25$, all combined $h_{S N V}^{2}=0.05$, $95 \%$ CI 0-0.12). As a result of the insufficient sample size, we could not estimate the $h_{S N V}^{2}$ of impairment/help-seeking in North America and diagnostic interview in Europe. For depression versus (hypo) manic and mixed PAO, $h_{S N V}^{2}$ was 0.17 (95\% CI 0.05-0.29) on the observed scale.

\section{Discussion}

In our study of bipolar disorder disease onset, we first evaluated the association between AAO or PAO with several clinical indicators of severity in a sample of 1659 patients. We showed that an earlier onset is associated with increased severity, demonstrating and replicating the clinical relevance of these phenotypes. Next, we performed genetic analyses including 12977 patients from 34 cohorts. Here, we demonstrated that higher genetic risk for ASD, major depression, schizophrenia and educational attainment is associated with an earlier AAO, providing evidence that the age at bipolar disorder onset is influenced by a broad liability for psychiatric illness.

Third, we performed GWAS to identify genetic variants associated with the AAO and PAO, which did not yield any replicated associations. Fourth, we outlined the extent to which age (and, partly, polarity) at onset varies across cohorts, depending both on the continent of recruitment and on the diagnostic instrument used to determine the AAO.

Finally, we showed that this substantial phenotypic heterogeneity affects the heritability of the phenotype, which decreased when multiple cohorts with different diagnostic instruments were combined. This analysis emphasises how genetic analyses are hampered by phenotypic heterogeneity.

\section{Illness onset is associated with disease course}

In a first set of analyses, we confirmed the clinical relevance of disease onset phenotypes in bipolar disorder. Age at bipolar disorder onset was associated with important illness severity indicators, such as suicidality, psychotic symptoms and lower educational attainment, thereby replicating findings of previous studies. $^{22,25}$ Furthermore, patients with a depressive bipolar disorder onset had an increased reported lifetime suicidality, whereas those with a (hypo)manic onset were more likely to experience delusions and more manic episodes per illness year. Contrary to previous evidence in a US (but not in a French) sample, we observed that an earlier onset was associated with fewer episodes per illness year. ${ }^{26}$ Of note, when not normalising for the illness duration, the AAO was, as expected, positively correlated with the number of episodes (see Supplementary Note 2).

\section{Increased genetic scores for neuropsychiatric phenotypes predict an earlier illness onset}

Higher PGSs for schizophrenia, major depression, ASD and educational attainment were significantly associated with a lower AAO, and none of the tested PGSs were significantly associated with PAO. Our findings support the hypothesis that a general liability for psychiatric disorders influences an earlier age of onset in bipolar disorder. Alternatively, an earlier onset may also reflect the broader phenotypic spectrum sometimes captured in earlyonset bipolar disorder. Unexpectedly, and in contrast to several other disorders (for example multiple sclerosis), where the strongest genetic risk factors for disease liability are also the most important genetic factors associated with an earlier disease onset, ${ }^{6,27}$ we did not find a significant association between bipolar disorder PGS and the age at bipolar disorder onset. Statistical power may have influenced this result, as the sample sizes of both the schizophrenia and major depression GWASs were larger than that of the bipolar disorder GWAS, improving the predictive ability of these PGSs compared with the bipolar disorder PGS.

The described significant relationship of higher educational attainment PGS with an earlier AAO may seem counterintuitive. However, several studies described a significant association, genetic correlation and causal relationship between a higher educational attainment and bipolar disorder risk. ${ }^{28,29}$ Our findings demonstrate that a high educational attainment PGS is not only a risk factor for bipolar disorder but also associated with an earlier onset of the disorder.

\section{Lack of replication of the GWAS finding}

We have conducted two GWASs to identify individual loci influencing the age and polarity at bipolar disorder onset, possibly independently of affecting lifetime disorder risk. Our discovery GWAS prioritised a genome-wide significant locus associated with the AAO. However, the lack of replication suggests that this finding may have been false-positive. This failure to replicate could have been because of insufficient statistical power in the replication sample, as our power analysis did not account for the likely phenotypic and genetic heterogeneity across cohorts and may thus have underestimated the necessary sample size. Importantly, the replication sample was more ethnically diverse than the discovery sample, which reduced the statistical power. The PAO GWAS, with its lower sample size and dichotomous phenotype, did not identify any genome-wide significant locus.

We also calculated an AAO PGS using our GWAS and tested it on our sample. Although the effect size of this PGS on the AAO was substantial ( 0.23 years per unit change in the PGS), the association was only nominally significant.

\section{The heterogeneity of phenotype definitions}

A striking finding of our study was the systematic difference in the AAO distribution across cohorts, continents and assessment strategies. Although the assessment strategies varied considerably by continent, with diagnostic interview being mainly used in North 
America and impairment/help-seeking in Europe, we showed that the continent-level differences were partially independent from the AAO assessment strategy and that both factors contributed significantly to the heterogeneity (Supplementary Table S6). However, variations in the demographic structure of analysed populations may have biased the assessed AAO of bipolar disorder, contributing to the observed differences. Although prior research has identified AAO differences across continents (for example the incidence of early-onset bipolar disorder is higher in the USA than in Europe $)^{30}$ this study is the first to systematically assess this heterogeneity across many cohorts with different ascertainment strategies.

For the polarity at disease onset, the relative proportion of patients reporting a depressive index episode did not differ across continents but across instruments. A (hypo)manic onset was more common if the onset was based on an impairment/helpseeking instead of diagnostic interview phenotype definition.

\section{Phenotypic heterogeneity affects genetic analyses}

Interestingly, the systematic differences in AAO phenotypes across cohorts are reflected in heritability estimates: we observed the highest SNV-based heritability $h_{S N V}^{2}$ when onset was established by diagnostic interview and the lowest when it was captured with more health system-specific and subjective measurements, such as item 4 of the Operational Criteria Checklist for Psychotic Illness (impairment/help-seeking). Moreover, $h_{S N V}^{2}$ estimates approached zero when all samples were combined in our primary analysis $\left(h_{S N V}^{2}=0.05 ; 95 \%\right.$ CI 0-0.12), underscoring the strong impact of phenotypic heterogeneity. For $\mathrm{PAO}-\mathrm{M} / \mathrm{X}$ versus $\mathrm{PAO}-\mathrm{D}$, we observed significant $h_{S N V}^{2}$ estimates, demonstrating that genetic factors contribute to the polarity at bipolar disorder onset.

Thus, we not only showed systematic heterogeneity in a clinically relevant psychiatric phenotype across cohorts but also provided direct evidence for how this heterogeneity can hamper genetic studies. Similarly, a recent investigation demonstrated that the phenotyping method (for example diagnostic interview versus self-report) significantly influenced heritability estimates, GWAS results and PGS performance in analyses of major depression susceptibility, with broader phenotype definitions resulting in lower heritability estimates. ${ }^{31}$ These results indicate that although increasing samples sizes generally improves the power to detect significant associations, larger samples are no silver bullet: careful phenotype harmonisation and uniform recruitment strategies are likely at least as important.

\section{Limitations}

In addition to diverse phenotype definitions originating from different ascertainment methods, as described above, several factors may have limited the cross-cohort comparability of the AAO and PAO. These factors include differences in the definition and ascertainment of the age at bipolar disorder onset and in how bipolar disorder was diagnosed across cohorts and continents. Such differences can lead to bias, affecting genetic analyses. For example, as patients diagnosed with bipolar disorder type II show, on average, later ages at onset than patients with bipolar disorder type $\mathrm{I},{ }^{32}$ differing proportions of bipolar disorder subtypes across cohorts may have an impact on AAO analyses. Therefore, we included the bipolar disorder subtype as a covariate in our genetic analyses to control for this confounder. Still, this cross-cohort heterogeneity has likely reduced our statistical power.

Given that, for all included cohorts, the disease onset phenotypes were assessed retrospectively, measurement errors associated with interrater reliabilities and recall bias may have occurred across cohorts. For example, hypomania was likely underreported, potentially biasing the PAO towards depression. Notably, such potential issues are not specific to the present study but may affect all retrospective analyses of psychiatric phenotypes. Nevertheless, differences in the diagnosis of bipolar disorder and the ascertained phenotypes between cohorts might have exacerbated these problems. Therefore, future studies should focus on compiling clinically more homogeneous, phenotypically better-harmonised data-sets instead of only assembling the largest possible sample.

Furthermore, the rank-based inverse normal transformation of the AAO phenotype may have affected the GWAS and heritability analyses. We conducted this transformation because, first, the original AAO distribution was highly skewed and thus not suitable for linear regression and, second, the AAO differed significantly between cohorts, which could have biased the meta-analysis. However, by transforming the data, only the rank and not the absolute differences in onset between patients was maintained, reducing the interpretability of the phenotype and the genetic effects.

We performed both SNV-level and polygenic score associations using a structured meta-analysis, which mitigates some of the noise introduced by phenotypic heterogeneity. However, we were unable to account for differences in the underlying genetic aetiology of the phenotypes across cohorts. As described above, phenotypic heterogeneity is an important limitation of our study and should be considered in future phenotype and genetic analyses. Our results need to be interpreted in light of these limitations.

\section{Implications}

Phenotypes of bipolar disorder onset are clinically important trait measures contributing to the well-known clinical and biological heterogeneity of this severe psychiatric disorder. Genetic analysis of AAO and PAO may lead to a better understanding of the biological risk factors underlying mental illness and support clinical assessment and prediction. Our study provides evidence of a genetic contribution to age and polarity at bipolar disorder onset but also demonstrates the need for systematic harmonisation of clinical data on bipolar disorder onset in future studies.

Janos L. Kalman (D), MD, Institute of Psychiatric Phenomics and Genomics (IPPG), University Hospital, LMU Munich, Germany; Department of Psychiatry and Psychotherapy, University Hospital Munich, Germany; and International Max Planck Research School for Translational Psychiatry, Germany; Loes M. Olde Loohuis, PhD, Center for Neurobehavioral Genetics, Semel Institute for Neuroscience and Human Behavior, University of California Los Angeles, USA; Annabel Vreeker, PhD, Department of Child and Adolescent Psychiatry/Psychology, Erasmus University Medical CentreSophia Children's Hospital, the Netherlands; Andrew McQuillin, PhD, Division of Psychiatry, University College London, UK; Eli A. Stahl, PhD, Division of Psychiatric Genomics, Mount Sinai School of Medicine, USA; Douglas Ruderfer, PhD, Division of Genetic Medicine, Department of Medicine, Vanderbilt Genetics Institute, Vanderbilt University Medical Center, USA; Department of Biomedical Informatics, Vanderbilt University Medical Center, USA; and Department of Psychiatry and Behavioral Sciences, Vanderbilt University Medical Center, USA; Maria Grigoroiu-Serbanescu, PhD, Alexandru Obregia Clinical Psychiatric Hospital, Bucharest, Romania;

Georgia Panagiotaropoulou, MSC, Department of Psychiatry and Psychotherapy,

Charite - Universitätsmedizin, Germany; Stephan Ripke, MD, PhD, Analytic and Translational Genetics Unit, Massachusetts General Hospital, USA; and Stanley Center for Psychiatric Research, Broad Institute of MIT and Harvard, USA; Tim B. Bigdeli, PhD, Department of Psychiatry and Behavioral Sciences, SUNY Downstate Health Sciences University, USA; and VA NY Harbor Healthcare System, USA; Frederike Stein, MA, Department of Psychiatry and Psychotherapy, Philipps-University Marburg, Germany; Tina Meller, Dr rer nat, MSc, Department of Psychiatry and Psychotherapy, PhilippsUniversity Marburg, Germany; and Center for Mind, Brain and Behavior (CMBB), Germany; Susanne Meinert, MSC, Institute for Translational Psychiatry, Westfälische WilhelmsUniversität Münster, Germany; and Institute for Translational Neuroscience, University of Münster, Germany; Helena Pelin, MSC, International Max Planck Research School for

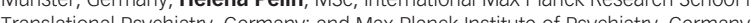
Translational Psychiatry, Germany; and Max Planck Institute of Psychiatry, Germany; of Mental Health, Medical Faculty Mannheim, Heidelberg University, Germany; Sergi Papiol, PhD, Institute of Psychiatric Phenomics and Genomics (IPPG), University Hospital, LMU Munich, Germany; Department of Psychiatry and Psychotherapy, University Hospital Munich, Germany; and Centro de Investigación Biomedica en Red de Salud Mental (CIBERSAM), Spain; Mark J. Adams (D), PhD, Division of Psychiatry, University of Edinburgh, UK; Rolf Adolfsson, MD, PhD, Department of Clinical Sciences, Medical Faculty, Umeå University, Sweden; Kristina Adorjan (D), MD, Institute of Psychiatric Phenomics and Genomics (IPPG), University Hospital, LMU Munich, Germany; and Department of Psychiatry and Psychotherapy, University Hospital Munich, Germany; Ingrid Agartz, MD, PhD, Department of Clinical Neuroscience, Centre for Psychiatry Research, Karolinska Institutet, Sweden; Department of Psychiatric Research, Diakonhjemmet Hospital, Norway; and NORMENT Centre, Division of Mental Health and 
Addiction, Institute of Clinical Medicine, University of Oslo, Norway; Sofie R. Aminoff PhD, Division of Mental Health and Addiction, Oslo University Hospital, Norway; and NORMENT Centre, Inst of Clinical Medicine, University of Oslo, Norway;

Heike Anderson-Schmidt, Dipl-Psych, Department of Psychiatry and Psychotherapy, University Medical Center Göttingen, Germany; Ole A. Andreassen, MD, PhD, NORMENT Centre, Inst of Clinical Medicine, University of Oslo, Norway; and Division of Mental Health and Addiction, Oslo University Hosptial, Norway; Raffaella Ardau, MD, Unit of Clinical Pharmacology, University Hospital Agency of Cagliari, Italy; JeanMichel Aubry, MD, Faculty of medicine, University of Geneva, Switzerland; and Department of Psychiatry, Psychosomatic Medicine and Psychotherapy, University Hospital Frankfurt, Germany; Ceylan Balaban, MScm, Department of Psychiatry, Psychosomatic Medicine and Psychotherapy, University Hospital Frankfurt, Germany; Nicholas Bass, MRCPsych, Division of Psychiatry, University College London, UK: Bernhard T. Baune, MD, PhD, Department of Psychiatry, University of Münster, Germany; Department of Psychiatry, Melbourne Medical School, The University of Melbourne, Australia; The Florey Institute of Neuroscience and Mental Health, The University of Melbourne, Australia; and Discipline of Psychiatry, Adelaide Medical School, The University of Adelaide, Australia; Frank Bellivier, MD, PhD, Universite de Paris, France; INSERM UMRS 1144, France; and DMU Neurosciences, GHU Lariboisière Fernand Widal, Departement de Psychiatrie, APHP, France; Antoni Benabarre, MD, PhD, Hospital Clinic, University of Barcelona, IDIBAPS, CIBERSAM, Spain;

Susanne Bengesser, MD, PhD, Department of Psychiatry and Psychotherapeutic Medicine, Medical University Graz, Austria; Wade H Berrettini, MD, PhD, Psychiatry, University of Pennsylvania, USA; Marco P. Boks, MD, PhD, Psychiatry, UMC Utrecht Brain Center, the Netherlands; Evelyn J. Bromet, PhD, Department of Psychiatry, Stony Brook University, USA; Katharina Brosch, MSC, Department of Psychiatry and Psychotherapy, Philipps-University Marburg, Germany; Monika Budde, PhD, Institute of Psychiatric Phenomics and Genomics (IPPG), University Hospital, LMU Munich, Germany; William Byerley, MD, Psychiatry, University of California San Francisco, USA; Pablo Cervantes, MD, Department of Psychiatry, McGill University, Canada; Catina Chillotti, MD, Unit of Clinical Pharmacology, University Hospital Agency of Cagliari, Italy; Sven Cichon, PhD, Department of Biomedicine, University of Basel, Switzerland; Institute of Human Genetics, University of Bonn, School of Medicine \& University Hospital Bonn, Germany; Institute of Medical Genetics and Pathology, University Hospital Basel, Switzerland; and Institute of Neuroscience and Medicine (INM1), Research Centre Julich, Germany; Scott R. Clark, MD, PhD, Discipline of Psychiatry, University of Adelaide, Australia; and Bazil Hetzel Institute, Australia; Ashley L. Comes, PhD, Institute of Psychiatric Phenomics and Genomics (IPPG), University Hospital, LMU Munich, Germany; Department of Psychiatry and Psychotherapy, University Hospital Munich, Germany; and International Max Planck Research School for Translational Psychiatry, Germany; Aiden Corvin, MD, PhD, Department of Psychiatry \& Trinity Translational Medicine Institute, Trinity College Dublin, Ireland; William Coryell, MD, University of lowa Hospitals and Clinics, USA; Nick Craddock, MD, Medical Research Council Centre for Neuropsychiatric Genetics and Genomics, Division of Psychological Medicine and Clinical Neurosciences, Cardiff University, UK; David W. Craig, PhD, Translational Genomics, USC, USA; Paul E. Croarkin, DO, MS, Department of Psychiatry and Psychology, Mayo Clinic, USA; Cristiana Cruceanu, PhD, Department of Translational Research, Max Planck Institute of Psychiatry, Germany; Piotr M. Czerski, PhD, Department of Psychiatric Genetics, Poznan University of Medical Sciences, Poland; Nina Dalkner, PhD, Department of Psychiatry and Psychotherapeutic Medicine, Medical University Graz, Austria; Udo Dannlowski, MD, PhD, Institute for Translational Psychiatry, Westfälische Wilhelms-Universität Münster, Germany;

Franziska Degenhardt, MD, Institute of Human Genetics, University of Bonn, School of Medicine \& University Hospital Bonn, Germany; and Department of Child and Adolescent Psychiatry, Psychosomatics and Psychotherapy, University Hospital Essen, University of Duisburg-Essen, Germany; Maria Del Zompo, MD, Department of Biomedical Science, Section of Neuroscience \& Clinical Pharmacology, University of Cagliari, Italy; and Unit of Clinical Pharmacology, University Hospital Agency of Cagliari, Italy;

J. Raymond DePaulo, MD, Department of Psychiatry and Behavioral Sciences, Johns Hopkins University, USA; Srdjan Djurovic, PhD, Department of Medical Genetics, Oslo University Hospital Ullevål, Norway; and NORMENT, Department of Clinical Science, University of Bergen, Norway; Howard J. Edenberg $\mathbb{D}$, PhD, Department of Biochemistry and Molecular Biology, Indiana University School of Medicine, USA: Mariam Al Eissa, PhD, Division of Psychiatry, University College London, UK; Torbjørn Elvsåshagen, $\mathrm{MD}, \mathrm{PhD}, \mathrm{NORMENT}$, Division of Mental Health and Addiction Oslo University Hospital, Norway; Bruno Etain (1D, MD, PhD, Universite de Paris, France INSERM UMRS 1144, France; and DMU Neurosciences, GHU Lariboisière Fernand Widal, Departement de Psychiatrie, APHP, France; Ayman H. Fanous, MD, Department of Psychiatry and Behavioral Sciences, SUNY Downstate Health Sciences University, USA; and VA NY Harbor Healthcare System, USA; Frederike Fellendorf, MD, Department of Psychiatry and Psychotherapeutic Medicine, Medical University Graz, Austria; Alessia Fiorentino, PhD, Division of Psychiatry, University College London, UK; Andreas J. Forstner, MD, Institute of Human Genetics, University of Bonn, School of Medicine \& University Hospital Bonn, Germany; and Centre for Human Genetics, University of Marburg, Germany; Mark A. Frye, MD, Department of Psychiatry and Psychology, Mayo Clinic, USA; Janice M. Fullerton, PhD, Neuroscience Research Australia, Australia; and School of Medical Sciences, University of New South Wales, Australia; Katrin Gade (D), MD, Department of Psychiatry and Psychotherapy, University Medical Center Göttingen, Germany; Julie Garnham, BN, RN, Nova Scotia Health Authority, Canada; Elliot Gershon, MD, Department of Psychiatry and Behaviora Neuroscience, University of Chicago, USA; and Department of Human Genetics, University of Chicago, USA; Michael Gill, MD, Department of Psychiatry \& Trinity Translational Medicine Institute, Trinity College Dublin, Ireland; Fernando S. Goes, MD, Department of Psychiatry and Behavioral Sciences, Johns Hopkins University, USA; Katherine Gordon-Smith (ID, PhD, Psychological Medicine, University of Worcester, UK; Paul Grof, MD, PhD, Mood Disorders Centre of Ottawa, Canada; and Department of Psychiatry, University of Toronto, Canada; Jose Guzman-Parra, PhD, Mental Health Department, University Regional Hospital, Biomedicine Institute (IBIMA), Spain; Tim Hahn, PhD, Institute for Translational Psychiatry, Westfälische Wilhelms-Universität Münster, Germany; Roland Hasler, PhD, Cell Biology, SUNY Downstate Medical Center College of Medicine, USA; and Institute for Genomic Health, SUNY Downstate Medical Center College of Medicine, USA; Maria Heilbronner, Dipl-Psych, Institute of Psychiatric Phenomics and Genomics (IPPG), University Hospital, LMU Munich, Germany;

Urs Heilbronner (ID), PhD, Institute of Psychiatric Phenomics and Genomics (IPPG),
University Hospital, LMU Munich, Germany; Stephane Jamain, PhD, Universite Paris Est Creteil, France; and INSERM U 955, Neuropsychiatrie Translationnelle, France: Esther Jimenez, MSC, PhD, Hospital Clinic, University of Barcelona, IDIBAPS, CIBERSAM, Spain; Ian Jones, PhD, MD, Medical Research Council Centre for Neuropsychiatric Genetics and Genomics, Division of Psychological Medicine and Clinical Neurosciences, Cardiff University, UK; Lisa Jones (1D), PhD, Psychological Medicine, University of Worcester, UK; Lina Jonsson, MSC, Department of Psychiatry and Neurochemistry Institute of Neuroscience and Physiology, The Sahlgrenska Academy at the University of Gothenburg, Sweden; Rene S. Kahn, MD, PhD, Department of Psychiatry, Icahn School of Medicine at Mount Sinai, USA; John R. Kelsoe, MD, Department of Psychiatry, University of California San Diego, USA; James L. Kennedy, MD, Department of Psychiatry, University of Toronto, Canada; The Campbell Family Mental Health Research Institute, Centre for Addiction and Mental Health, Canada; and Institute of Medical Science, University of Toronto, Canada; Tilo Kircher, MD, Department of Psychiatry and Psychotherapy, Philipps-University Marburg, Germany; George Kirov, PhD, MD, Medical Research Council Centre for Neuropsychiatric Genetics and Genomics, Division of Psychological Medicine and Clinical Neurosciences, Cardiff University, UK; Sarah KittelSchneider, MD, Department of Psychiatry, Psychosomatic Medicine and Psychotherapy, University Hospital Frankfurt, Germany; and Department of Psychiatry, Psychotherapy and Psychosomatics, University Hospital Wurzburg, Germany: Farah Klöhn-Saghatolislam, MD, MBA, Institute of Psychiatric Phenomics and Genomics (IPPG), University Hospital, LMU Munich, Germany; James A. Knowles, MD, PhD, Cell Biology, SUNY Downstate Medical Center College of Medicine, USA; and Institute for Genomic Health, SUNY Downstate Medical Center College of Medicine, USA; Thorsten M. Kranz, PhD, Department of Psychiatry, Psychosomatic Medicine and Psychotherapy, University Hospital Frankfurt, Germany; Trine Vik Lagerberg, PhD, NORMENT Centre, Division of Mental Health and Addiction, Oslo University Hosptial, Norway; Mikael Landen, MD, PhD, Department of Psychiatry and Neurochemistry, Institute of Neuroscience and Physiology, The Sahlgrenska Academy at the University of Gothenburg, Sweden; and Department of Medical Epidemiology and Biostatistics, Karolinska Institutet, Sweden; William B. Lawson, MD, Department of Psychiatry and Behavioral Sciences, Howard University Hospital, USA; Marion Leboyer, MD, PhD, Universite Paris Est Creteil, France; and INSERM U 955, Neuropsychiatrie Translationnelle, France; Qingqin S. Li, PhD, Neuroscience, Janssen Research \& Development, USA; Mario Maj, MD, PhD, Department of Psychiatry, University of Campania 'Luigi Vanvitell', Italy; Dolores Malaspina, MD, MS, MSPH, Department of Psychiatry, Icahn School of Medicine at Mount Sinai, USA; and Department of Genetics \& Genomics, Icahn School of Medicine at Mount Sinai, USA; Mirko Manchia, MD, PhD, Unit of Psychiatry, Department of Medical Sciences and Public Health, University of Cagliari, Italy and Department of Pharmacology, Dalhousie University, Canada; Fermin Mayoral, PhD, MDF, Mental Health Department, University Regional Hospital, Biomedicine Institute (IBIMA), Spain; Susan L. McElroy, MD, Research Institute, Lindner Center of HOPE, USA: Melvin G. McInnis, MD, Department of Psychiatry, University of Michigan, USA: Andrew M. McIntosh (D, MD, FRCPsych, Division of Psychiatry, University of Edinburgh, UK; Helena Medeiros, MSW, LICSW, Institute for Genomic Health, SUNY Downstate Medical Center College of Medicine, USA; Ingrid Melle, MD, PhD, NORMENT Centre, Division of Mental Health and Addiction, Institute of Clinical Medicine and Diakonhjemmet Hospital, University of Oslo, Norway; and Division of Mental Health and Addiction, Oslo University Hospital, Norway; Vihra Milanova, MD, PhD, Psychiatric Clinic, Alexander University Hospital, Bulgaria; Philip B. Mitchell (D, MD, School of Psychiatry, University of New South Wales, Australia; Palmiero Monteleone, MD, Department of Medicine, Surgery and Dentistry 'Scuola Medica Salernitana', University of Salerno, Italy; Alessio Maria Monteleone, MD, Department of Psychiatry, University of Campania 'Luigi Vanvitelli', Italy; Markus M. Nöthen, MD, Institute of Human Genetics, University of Bonn, School of Medicine \& University Hospital Bonn, Germany; Tomas Novak, PhD, National Institute of Mental Health, Czech Republic; John I. Nurnberger (D, MD, PhD, Psychiatry, Indiana University school of Medicine, USA; Niamh O'Brien, PhD, Division of Psychiatry, University College London, UK; Kevin S. O'Connell, PhD, Division of Mental Health and Addiction, Oslo University Hospital, Norway; and NORMENT Centre, Inst of Clinical Medicine, University of Oslo, Norway; Claire O'Donovan, MB, BchBAO, Department of Psychiatry, Dalhousie University, Canada; Michael C. O'Donovan, PhD, MD, Medical Research Council Centre for Neuropsychiatric Genetics and Genomics, Division of Psychological Medicine and Clinical Neurosciences, Cardiff University, UK; Nils Opel, MD, Institute for Translational Psychiatry, Westfälische Wilhelms-Universität Münster, Germany; Abigail ortiz $\mathbb{D}, \mathrm{MD}$, MSC, FRCPC, Department of Psychiatry, University of Toronto, Toronto, Canada; and Centre for Addiction and Mental Health, Toronto, Canada; Michael J. Owen, PhD, MD, Medical Research Council Centre for Neuropsychiatric Genetics and Genomics, Division of Psychological Medicine and Clinical Neurosciences, Cardiff University, UK; Erik Pålsson, PhD, Department of Psychiatry and Neurochemistry, Institute of Neuroscience and Physiology, The Sahlgrenska Academy at the University of Gothenburg, Sweden; Carlos Pato, MD, PhD, Institute for Genomic Health, SUNY Downstate Medical Center College of Medicine, USA; Michele T. Pato, MD, Institute for Genomic Health, SUNY Downstate Medical Center College of Medicine, USA; Joanna Pawlak, PhD, MD, Department of Psychiatric Genetics, Poznan University of Medical Sciences, Poland; Julia-Katharina Pfarr, MSC, Department of Psychiatry and Psychotherapy, Philipps-University Marburg, Germany; Claudia Pisanu, MD, Department of Biomedical Science, Section of Neuroscience \& Clinical Pharmacology, University of Cagliari, Italy; James B. Potash, MD, Department of Psychiatry and Behavioral Sciences, Johns Hopkins University, USA; Mark H Rapaport, MD, Department of Psychiatry and Behavioral Sciences, Emory University, USA; Daniela Reich-Erkelenz, MA, Institute of Psychiatric Phenomics and Genomics (IPPG), University Hospital, LMU Munich, Germany; Andreas Reif, MD, Department of Psychiatry, Psychosomatic Medicine and Psychotherapy, University Hospital Frankfurt Germany: Eva Reininghaus, MD, PhD, Department of Psychiatry and Psychotherapeutic Medicine, Medical University Graz, Austria; Jonathan Repple, MD, Institute for Translational Psychiatry, Westfälische Wilhelms-Universität Münster, Germany; Hélène Richard-Lepouriel, MD, Department of Psychiatry, Geneva University Hospitals, Switzerland; Marcella Rietschel, MD, Department of Genetic Epidemiology in Psychiatry, Central Institute of Mental Health, Medical Faculty Mannheim, Heidelberg University, Germany; Kai Ringwald, MSc, Department of Psychiatry and Psychotherapy, Philipps-University Marburg, Germany; Gloria Roberts (D), PhD, School of Psychiatry, University of New South Wales, Australia; Guy Rouleau, MD, PhD, FRCPC, FRSC Montreal Neurological Institute, Canada and Department of Neurology, McGill University, 
Canada; Sabrina Schaupp, MSc, Institute of Psychiatric Phenomics and Genomics (IPPG), University Hospital, LMU Munich, Germany; William A Scheftner, MD, Department of Psychiatry, Rush Medical College, USA; Simon Schmitt, MSC, Department of Psychiatry and Psychotherapy, Philipps-University Marburg, Germany; Peter R. Schofield, PhD DSC, Neuroscience Research Australia, Australia; and School o Medical Sciences, University of New South Wales, Australia; K. Oliver Schubert, MD, PhD, Discipline of Psychiatry, University of Adelaide, Australia; and Northern Adelaide Mental Health Service, SA Health, Australia; Eva C. Schulte (D), MD, PhD, Institute of Psychiatric Phenomics and Genomics (IPPG), University Hospital, LMU Munich, Germany; and Department of Psychiatry and Psychotherapy, University Hospital Munich, Germany; Barbara Schweizer, RN, BS, Department of Psychiatry and Behavioral Sciences, John Hopkins University, USA; Fanny Senner, MD, Institute of Psychiatric Phenomics and Genomics (IPPG), University Hospital, LMU Munich, Germany; and Department of Psychiatry and Psychotherapy, University Hospital Munich, Germany;

Giovanni Severino, MD, Department of Biomedical Science, Section of Neuroscience \& Clinical Pharmacology, University of Cagliari, Italy; Sally Sharp, PhD, Division of Psychiatry, University College London, UK; Claire Slaney, RN, Nova Scotia Health Authority, Canada; Olav B. Smeland, MD, PhD, Division of Mental Health and Addiction, Oslo University Hospital, Norway; and NORMENT Centre, Inst of Clinical Medicine, University of Oslo, Norway; Janet L. Sobell, PhD, Psychiatry and the Behavioral Sciences, University of Southern California, USA; Alessio Squassina, Ph.D. MSci, Department of Psychiatry, Dalhousie University, Canada; and Department of Biomedica Science, Section of Neuroscience \& Clinical Pharmacology, University of Cagliari, Italy; Pavla Stopkova, PhD, National Institute of Mental Health, Czech Republic; John Strauss, MD, Department of Psychiatry, University of Toronto, Canada; The Campbell Family Mental Health Research Institute, Centre for Addiction and Mental Health, Canada; and Institute of Medical Science, University of Toronto, Canada; Alfonso Tortorella, PhD, Department of Psychiatry, University of Perugia, Italy: Gustavo Turecki, MD, PhD, Department of Psychiatry, McGill University, Canada; and Douglas Institute, McGill University, Canada; Joanna Twarowska-Hauser, MD, PhD, Department of Psychiatric Genetics, Poznan University of Medical Sciences, Poland; Marin Veldic, MD, Department of Psychiatry and Psychology, Mayo Clinic, USA; Eduard Vieta, MD, PhD, Hospital Clinic, University of Barcelona, IDIBAPS, CIBERSAM, Spain; John B. Vincent, PhD, Department of Psychiatry, University of Toronto, Canada; The Campbell Family Mental Health Research Institute, Centre for Addiction and Mental Health, Canada; and Institute of Medical Science, University of Toronto, Canada; Wei Xu $\mathrm{PhD}$, Dalla Lana School of Public Health, Biostatistics Division, University of Toronto, Canada; Clement C. Zai, PhD, Department of Psychiatry, University of Toronto, Canada; The Campbell Family Mental Health Research Institute, Centre for Addiction and Menta Health, Canada; Institute of Medical Science, University of Toronto, Canada; Laboratory Medicine and Pathobiology, University of Toronto, Canada; and Harvard T.H. Chan Schoo of Public Health, USA; Peter P. Zandi, MHS, MPH, PhD, Department of Psychiatry and Behavioral Sciences, Johns Hopkins University, USA; Psychiatric Genomics Consortium (PGC) Bipolar Disorder Working Group; International Consortium on Lithium Genetics (ConLiGen); Colombia-US Cross Disorder Collaboration in Psychiatric Genetics; Arianna Di Florio, MD, PhD, MD, Medical Research Council Centre for Neuropsychiatric Genetics and Genomics, Division of Psychological Medicine and Clinical Neurosciences, Cardiff University, UK; Jordan W. Smoller, MD, SCD, Psychiatric and Neurodevelopmental Genetics Unit, Department of Psychiatry and Center for Genomic Medicine, Massachusetts General Hospital, USA; and Stanley Center for Psychiatric Research, Broad Institute of MIT and Harvard, USA; Joanna M. Biernacka, PhD, Department of Psychiatry and Psychology, Mayo Clinic, USA; and Department of Health Sciences Research, Mayo Clinic, USA; Francis J. McMahon, MD Human Genetics Branch, Intramural Research Program, National Institute of Mental Health, USA; Martin Alda (D), MD, National Institute of Mental Health, Czech Republic and Department of Psychiatry, Dalhousie University, Canada; Bertram Müller-Myhsok MD, Max Planck Institute of Psychiatry, Germany; Nikolaos Koutsouleris, MD, Department of Psychiatry and Psychotherapy, University Hospital Munich, Germany; Max Planck Institute of Psychiatry, Germany; and Institute of Psychiatry, Psychology and Neuroscience, Kings College London, UK; Peter Falkai, MD, Department of Psychiatry and Psychotherapy, University Hospital Munich, Germany; Nelson B. Freimer, MD, Center for Neurobehavioral Genetics, Semel Institute for Neuroscience and Human Behavior, University of California Los Angeles, USA; and Human Genetics, University of California Los Angeles, USA; Till F.M. Andlauer (D), PhD, Department of Neurology, Klinikum rechts der Isar, School of Medicine, Technical University of Munich, Germany; Thomas G. Schulze, MD, Institute of Psychiatric Phenomics and Genomics (IPPG), University Hospital, LMU Munich, Germany; Department of Genetic Epidemiology in Psychiatry, Central Institute of Mental Health, Medical Faculty Mannheim, Heidelberg University, Germany; Department of Psychiatry and Psychotherapy, University Medical Center Göttingen, Germany; Department of Psychiatry and Behavioral Sciences, Johns Hopkins University School of Medicine, USA; and Department of Psychiatry and Behavioral Sciences, SUNY Upstate Medical University, USA; Roel A. Ophoff, PhD, Center for Neurobehavioral Genetics, Semel Institute for Neuroscience and Human Behavior, University of California Los Angeles, USA; Human Genetics, University of California Los Angeles, USA; and Psychiatry, Erasmus University Medical Center, the Netherlands

Correspondence: Janos L. Kalman. Email: janos.kalman@med.uni-muenchen.de; Loes M. Olde Loohuis. Email: Ioldeloohuis@mednet.ucla.edu

First received 11 Dec 2020, final revision 26 May 2021, accepted 1 Jul 2021

\section{Supplementary material}

To view supplementary material for this article, please visit https://doi.org/10.1192/bjp.2021.102

\section{Data availability}

The data that support the findings of this study are available from the corresponding author, upon reasonable request.

\section{Acknowledgments}

The authors thank Jacquie Klesing, Board-certified Editor in the Life Sciences (ELS), for editing assistance with the manuscript BOMA-Australia sample: we thank Gin Mahli, Colleen Loo, and Micheal Breaskpear for their contribution to clinical assessments of a subset of patients and also Andrew Frankland for his work in collating clinical record data. WTCCC sample: this study makes use of data generated by the Wellcome Trust Case-Control Consortium. A full list of the investigators who contributed to the generation of the data is available from www. wtccc.org.uk. French sample: we thank the psychiatrists and psychologists who participated in the clinical assessment of patients in France (C. Henry, S. Gard, J.P. Kahn, L. Zanouy, R.F. Cohen and O. Wajsbrot-Elgrabli) and thank the patients for their participation.

\section{Author contributions}

Concept and design: Janos L. Kalman, Loes M. Olde Loohuis, Annabel Vreeker, Till F.M. Andlauer, Thomas G. Schulze and Roel A. Ophoff. Analysis and interpretation of data: Janos L Kalman, Loes M. Olde Loohuis, Annabel Vreeker and Till F.M. Andlauer. Drafting of the manuscript: Janos L. Kalman, Loes M. Olde Loohuis, Annabel Vreeker and Till F.M. Andlauer. Supervision, and critical revision of the manuscript: Thomas G. Schulze, Roel A. Ophoff, Francis J. McMahon, Jordan W. Smoller and Martin Alda. All other authors provided data, contributed ideas and suggestions for analyses, interpreted results and revised the final manuscript.

\section{Funding}

The funding details can be found in the Supplementary File 1

\section{Declaration of interest}

Amare T. Azmeraw: has received 2020-2022 NARSAD Young Investigator Grant from the Brain \& Behaviour Research Foundation. Ole A. Andreassen: speaker's honorarium Sunovion, Lundbeck. Consultant HealthLytix. Bernhard Baune: Honoraria: Lundbeck, Janssen, LivaNova, Servier. Carrie Bearden: Novartis Scientific Advisory Board. Clark Scott: Honoraria and Investigator-initiated project funding from Jannsen-Cilag Australia, Lundbeck-Otsuka Australia. J. Raymond DePaulo: owns stock in CVS Health; JRD was unpaid consultant for Myriad Neuroscience 2017 \& 2019. Michael C. O'Donovan: research unrelated to this manuscript supported by a collaborative research grant from Takeda Pharmaceuticals. Bruno Etain: honoraria for Sanofi. Mark A. Frye: grant Support Assurex Health, Mayo Foundation, Medibio Consultant (Mayo) Actify Neurotherapies, Allergan, Intra-Cellular Therapies, Inc., Janssen, Myriad, Neuralstem Inc., Sanofi, Takeda, Teva Pharmaceuticals. Per Hoffmann: employee of Life\&Brain GmbH, Member of the Scientific Advisory Board of HMG Systems Engeneering $\mathrm{GmbH}$. Mikael Landen: Speaker's honoraria Lundbeck pharmaceuticals. Andrew M McIntosh: research funding from The Sackler Trust, speaker fees from Illumina and Janssen. Philip B. Mitchell: remuneration for lectures in China on bipolar disorder research by Sanofi (Hangzhou). John Nurnberger: investigator for Janssen. Benjamin M. Neale: is a member of the scientific advisory board at Deep Genomics and RBNC Therapeutics. A consultant for Camp4 Therapeutics, Takeda Pharmaceutical and Biogen. Andreas Reif: apeaker's honoraria / Advisory boards: Janssen, Shire/Takeda, Medice, SAGE and Servier. Eli Stahl: now employed by the Regeneron Genetics Center. Kato Tadafumi: honoraria: Kyowa Hakko Kirin Co., Ltd., Eli Lilly Japan K.K., Otsuka Pharmaceutical Co., Ltd., GlaxoSmithKline K.K., Taisho Pharma Co., Ltd., Taisho Pharmaceutical Co, Ltd. Taisho Toyama Pharmaceutical Co. Ltd., Dainippon Sumitomo Pharma Co., Ltd., Meiji Seika Pharma Co., Ltd., Pfizer Japan Inc., Mochida Pharmaceutical Co., Ltd., Shionogi \& Co., Ltd., Janssen Pharmaceutical K.K., Janssen Asia Pacific, Yoshitomiyakuhin, Astellas Pharma Inc., Nippon, Boehringer Ingelheim Co. Ltd., MSD K.K., Kyowa Pharmaceutical Industry Co., Ltd., Takeda Pharmaceutical Co., Ltd., Mitsubish Tanabe Pharma Corporation, Eisai Co., Ltd. Grants: Takeda Pharmaceutical Co., Ltd. Dainippon Sumitomo Pharma Co., Ltd., Otsuka Pharmaceutical Co., Ltd., Shionogi \& Co., Ltd. Eisai Co, Ltd Mitsubishi Tanabe Pharma Corporation. Thomas G. Schulze is a member of the editorial board of The British Journal of Psychiatry. He did not take part in the review or decision-making process of this paper. Eduard Vieta: has received grants and served as consultant, advisor or CME speaker for the following entities: AB-Biotics, Abbott, Allergan, Angelini, Dainippon Sumitomo Pharma, Ferrer, Gedeon Richter, Janssen, Lundbeck, Otsuka, Sage, Sanofi-Aventis, Sunovion and Takeda. None of the other authors reported any biomedical financial interests or potential conflicts of interest. ICMJE forms are in the supplementary material, available online at https://doi.org/10.1192/10.1192/bjp.2021.102.

\section{References}

1 Cloutier M, Greene M, Guerin A, Touya M, Wu E. The economic burden of bipolar I disorder in the United States in 2015. J Affect Disord 2018; 226: 45-51.

2 Berk M, Dodd S, Callaly P, Berk L, Fitzgerald P, de Castella AR, et al. History of illness prior to a diagnosis of bipolar disorder or schizoaffective disorder. J Affect Disord 2007 Nov; 103: 181-6.

3 Dagani J, Signorini G, Nielssen O, Bani M, Pastore A, Girolamo Gd, et al. Metaanalysis of the Interval between the Onset and Management of Bipolar Disorder. Can J Psychiatry 2017; 62: 247-58.

4 Cleynen I, Boucher G, Jostins L, Schumm LP, Zeissig S, Ahmad T, et al. Inherited determinants of Crohn's disease and ulcerative colitis phenotypes: A genetic association study. Lancet 2016; 387: 156-67.

5 Bergen SE, O'Dushlaine CT, Lee PH, Fanous AH, Ruderfer DM, Ripke S, et al Genetic modifiers and subtypes in schizophrenia: Investigations of age at onset, severity, sex and family history. Schizophr Res 2014; 154: 48-53. 
6 Naj AC, Jun G, Reitz C, Kunkle BW, Perry W, Park YS, et al. Effects of multiple genetic loci on age at onset in late-onset Alzheimer disease: A genome-wide association study. JAMA Neurol 2014; 71: 1394-404.

7 Rhebergen D, Lamers F, Spijker J, De Graaf R, Beekman ATF, Penninx BWJH. Course trajectories of unipolar depressive disorders identified by latent class growth analysis. Psychol Med 2012; 42: 1383-96.

8 Howes OD, Lim S, Theologos G, Yung AR, Goodwin GM, McGuire P. A com prehensive review and model of putative prodromal features of bipolar affective disorder. Psychol Med 2011; 41: 1567-77.

9 Wijnands JMA, Kingwell E, Zhu F, Zhao Y, Högg T, Stadnyk K, et al. Health-care use before a first demyelinating event suggestive of a multiple sclerosis prodrome: a matched cohort study. Lancet Neurol 2017 Jun 1; 16: 445-51.

10 Smoller JW, Finn CT. Family, twin, and adoption studies of bipolar disorder. Am J Med Genet Part C Semin Med Genet 2003 Nov 15; 123C: 48-58.

11 Rice J, Reich T, Andreasen NC, Endicott J, Van Eerdewegh M, Fishman R, et al. The familial transmission of bipolar illness. Arch Gen Psychiatry 1987; 44: 441-7.

12 Hafeman DM, Merranko J, Goldstein TR, Axelson D, Goldstein BI, Monk K, et al. Assessment of a person-level risk calculator to predict new-onset bipolar spectrum disorder in youth at familial risk. JAMA Psychiatry 2017 Aug 1 74: 841 .

13 Ruderfer DM, Ripke S, McQuillin A, Boocock J, Stahl EA, Pavlides JMW, et al. Genomic dissection of bipolar disorder and schizophrenia, including 28 subphenotypes. Cell 2018; 1737: 1705-1715.e16.

14 Stahl EA, Breen G, Forstner AJ, McQuillin A, Ripke S, Trubetskoy V, et al. Genome-wide association study identifies 30 loci associated with bipolar disorder. Nat Genet 2019; 51: 793-803.

15 Coleman JRI, Gaspar HA, Bryois J, Byrne EM, Forstner AJ, Holmans PA, et al. The genetics of the mood disorder spectrum: genome-wide association analyse of more than 185,000 cases and 439,000 controls. Biol Psychiatry 2020; 88(2): 169-184

16 Kalman JL, Papiol S, Forstner AJ, Heilbronner U, Degenhardt F, Strohmaier J, et al. Investigating polygenic burden in age at disease onset in bipolar disorder: Findings from an international multicentric study. Bipolar Disord 2019 Jun 28: 21: $68-75$.

17 Aas M, Bellivier F, Bettella F, Henry C, Gard S, Kahn JP, et al. Childhood maltreatment and polygenic risk in bipolar disorders. Bipolar Disord 2020 22: 174-81.

18 Belmonte Mahon P, Pirooznia M, Goes FS, Seifuddin F, Steele J, Lee PH, et al. Genome-wide association analysis of age at onset and psychotic symptoms in bipolar disorder. Am J Med Genet B Neuropsychiatr Genet 2011 Apr; 156B 370-8.

19 Jamain S, Cichon S, Etain B, Mühleisen TW, Georgi A, Zidane N, et al. Common and rare variant analysis in early-onset bipolar disorder vulnerability. PLOS One 2014; 9: e104326.

20 Kassem L, Lopez V, Hedeker D, Steele J, Zandi P, Bipolar Disorder Consortium $\mathrm{NGl}$, et al. Familiality of polarity at illness onset in bipolar affective disorder. Am J Psychiatry 2006 Oct; 163: 1754-9.
21 Schulze TG, Alda M, Adli M, Akula N, Ardau R, Bui ET, et al. The International Consortium on Lithium Genetics (ConLiGen): an initiative by the NIMH and IGSLI to study the genetic basis of response to lithium treatment Neuropsychobiology 2010; 62: 72-8.

22 Van Bergen AH, Verkooijen S, Vreeker A, Abramovic L, Hillegers MH, Spijker AT, et al. The characteristics of psychotic features in bipolar disorder. Psychol Med 2019 Sep 10; 49: 2036-48.

23 Budde $\mathrm{M}$, Anderson-Schmidt $\mathrm{H}$, Gade K, Reich-Erkelenz D, Adorjan K, Kalman $\mathrm{JL}$, et al. A longitudinal approach to biological psychiatric research: the PsyCourse study. Am J Med Genet B Neuropsychiatr Genet 2019; 180: 89-102.

24 Kircher T, Wöhr M, Nenadic I, Schwarting R, Schratt G, Alferink J, et al. Neurobiology of the major psychoses: a translational perspective on brain structure and function-the FOR2107 consortium. Eur Arch Psychiatry Clin Neurosci 2019: 269: 949-62.

25 Perlis RH, Miyahara S, Marangell LB, Wisniewski SR, Ostacher M, DelBello MP, et al. Long-term implications of early onset in bipolar disorder: data from the first 1000 participants in the systematic treatment enhancement program for bipolar disorder (STEP-BD). Biol Psychiatry 2004 May 1; 55: 875-81.

26 Etain B, Lajnef M, Bellivier F, Mathieu F, Raust A, Cochet B, et al. Clinical expression of bipolar disorder type I as a function of age and polarity at onset: Convergent findings in samples from France and the United States. J Clin Psychiatry 2012; 73(4): e561-6.

27 Andlauer TFM, Buck D, Antony G, Bayas A, Bechmann L, Berthele A, et al. Nove multiple sclerosis susceptibility loci implicated in epigenetic regulation. Sci Adv 2016 Jun 17; 2: e1501678.

28 Mullins N, Forstner AJ, O'Connell KS, Coombes B, Coleman JRI, Qiao Z, et al. Genome-wide association study of over 40,000 bipolar disorder cases provides novel biological insights. medRxiv [Preprint]. 2020; Available from: https://www.medrxiv.org/content/10.1101/2020.09.17.20187054v1.

29 Vreeker A, Boks MPM, Abramovic L, Verkooijen S, Van Bergen AH, Hillegers $\mathrm{MHJ}$, et al. High educational performance is a distinctive feature of bipolar disorder: a study on cognition in bipolar disorder, schizophrenia patients, relatives and controls. Psychol Med 2016 Mar 1; 46: 807-18.

30 Post RM, Luckenbaugh DA, Leverich GS, Altshuler LL, Frye MA, Suppes T, et al. Incidence of childhood-onset bipolar illness in the USA and Europe. $\mathrm{Br} J$ Psychiatry 2008 Feb; 192: 150-1.

31 Cai N, Revez JA, Adams MJ, Andlauer TFM, Breen G, Byrne EM, et al. Minimal phenotyping yields genome-wide association signals of low specificity for major depression. Nat Genet 2020; 52: 437-47.

32 Manchia M, Maina G, Carpiniello B, Pinna F, Steardo L, D'Ambrosio V, et al. Clinical correlates of age at onset distribution in bipolar disorder: a comparison between diagnostic subgroups. Int J Bipolar Disord 2017 Dec 1; 5: 28.

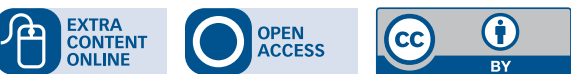

